\title{
Habilidades Matemáticas em Pessoas com Deficiência Intelectual: um Olhar Sobre os Estudos Experimentais ${ }^{1}$
}

\author{
MATHEMATICAL SKILLS IN PEOPLE WITH INTELLECTUAL DISABILITIES: AN OVERVIEW \\ OVER EXPERIMENTAL STUDIES
}

\author{
Ailton Barcelos da COSTA ${ }^{2}$ \\ Alessandra Daniele Messali PICHARILLO ${ }^{3}$ \\ Nassim Chamel ELIAS ${ }^{4}$
}

\begin{abstract}
RESUMO: o conhecimento das habilidades matemáticas é considerado fundamental para pessoas terem uma vida independente, mas de difícil aprendizado para qualquer um, inclusive para pessoas com deficiência intelectual, caracterizadas por terem déficits em determinadas habilidades gerais. Estas pessoas apresentam dificuldades nas habilidades que requeiram atenção, memória, raciocínio, generalização e abstração, que são fundamentais para o aprendizado acadêmico. Estudos sobre habilidades matemáticas, tanto para pessoas com deficiência intelectual como aquelas com desenvolvimento típico, apontam a inexistência de avaliação dessas habilidades, sob o ponto de vista contínuo (como medidas e geometria), mas por outro lado, é bastante forte em relação aos aspectos discretos da matemática (como contagem, aritmética e numeração). Dessa forma, esta pesquisa buscou identificar estratégias, conteúdos ensinados e o repertório de entrada para ensino de matemática a pessoas com deficiência intelectual, priorizando as pesquisas empíricas, presentes na literatura. A revisão sistema em base dados identificou 15 artigos que atendiam aos critérios almejados. O tratamento de dados indicou três temas de análise: conteúdo matemático, repertório de entrada e estratégias de ensino. Como resultado, conclui-se que há um número reduzido de trabalhos sobre do ensino de matemática a pessoas com deficiência intelectual. Também, percebe-se que há ainda um longo caminho a ser percorrido, em busca de uma escola inclusiva, na qual estas pessoas tenham acesso à matemática elementar.
\end{abstract}

PALAVRAS-CHAVE: Educação Especial. Ensino de Matemática. Deficiência Intelectual.

ABSTRACT: Knowledge in mathematics skills is considered essential for people to have an independent life, but difficult to learn for anyone, including people with intellectual disabilities, characterized by having deficits in certain general skills. These people have difficulties in skills requiring attention, memory, reasoning, generalization and abstraction, which are fundamental to academic learning. Studies on math skills, both for people with intellectual disabilities as those with typical development, point to the lack of assessment of these skills under continuous point of view (as measurements and geometry), but on the other hand, it is strong enough regarding discrete aspects of mathematics (such as counting, arithmetic and numbers). Thus, this research aimed to identify strategies, taught content, and the input repertoire for teaching math to people with intellectual disabilities, giving priority to empirical research in the literature. As a result, the review in the database 15 articles that met the criteria. The data indicated three themes for analysis: math content, input repertoire and teaching strategies. It is concluded that there is a reduced number of studies on the teaching of mathematics to people with intellectual disabilities. In addition, it is clear that there is still a long way to go in the search of an inclusive school, where these people have access to elementary mathematics.

KEYWORDS: Special Education. Mathematics Teaching. Intellectual Disabilities.

\footnotetext{
${ }^{1}$ http://dx.doi.org/10.1590/S1413-65382216000100011

${ }^{2}$ Doutorando do Programa de Pós-Graduação em Educação Especial da Universidade Federal de São Carlos, São Carlos, SP, Brasil. ailton_barcelos@yahoo.com.br

${ }^{3}$ Licencianda do Curso de Licenciatura em Educaçấo Especial da Universidade Federal de São Carlos, São Carlos, SP, Brasil. alessandrapicharillo@outlook.com

${ }^{4}$ Doutor em Educação Especial, Docente do Programa de Pós-Graduação em Educação Especial da Universidade Federal de São Carlos, São Carlos, SP, Brasil. nchamel@terra.com.br
} 


\section{INTRODUÇÁo}

A matemática está entrelaçada com as necessidades da vida moderna, sendo fundamental para uma vida independente, porém, de 5\% a 7\% de toda a população mundial têm sérias dificuldades com o aprendizado dessas habilidades (BRANKAE; GHESQUIÈRE; DE SMEDT, 2013). Já no Brasil, os dados não são muito alentadores, já que 67,5\% dos alunos na faixa etária de 10 anos ( $5^{\circ}$ ano do Ensino Fundamental) apresentaram dificuldades na aprendizagem deste conteúdo, não atingindo o aprendizado adequado para esta disciplina (CRUZ; BERGAMASCHI; REIS, 2012). Tais dados evidenciam a matemática como uma disciplina de difícil aprendizado, devido à grande abstração de seus conceitos, para qualquer um, não sendo diferente para pessoas com capacidade intelectual abaixo da média (BRANKAER; GHESQUIÈRE; DE SMEDT, 2013).

Dessa forma, pode-se dizer que a atual definição proposta pela American Psychiatric Association (APA, 2013), caracteriza a pessoa com deficiência intelectual por déficits de habilidades mentais gerais, tais como raciocínio, resolução de problemas, planejamento, pensamento abstrato, julgamento, aprendizagem escolar e de aprendizagem a partir da experiência. $\mathrm{O}$ déficit resulta em prejuízo no funcionamento adaptativo, de modo que o indivíduo não cumpra as normas de independência pessoal e responsabilidade social em um ou mais aspectos da vida diária, incluindo comunicação, participação social, funcionamento escolar ou profissional, pessoal e independência, em casa ou em ambientes comunitários (APA, 2013).

Os alunos com deficiência intelectual apresentam dificuldades em habilidades fundamentais para o aprendizado acadêmico em geral, como citado abaixo (CHUNG; TAM, 2005; MALAQUIAS at. al., 2013):

- Habilidades de percepção: estes alunos têm dificuldade com as relaçóes espaciais, distâncias, e sequenciamento, que podem interferir na aquisição de conceitos e habilidades matemáticos.

- Pensamento e raciocínio: estes estudantes têm dificuldades no pensamento e no raciocínio, levando a dificuldades na resolução de problemas.

- Memória: alguns destes alunos têm dificuldades em lembrar-se de informaçóes que foram apresentadas, principalmente símbolos abstratos usados em matemática (menos, maior que, menor que, etc.).

- Generalização: para tais alunos, a generalização é muitas vezes um grande obstáculo para a aprendizagem de matemática, pois sua aprendizagem tende a ser altamente específica, e o que se aprendeu raramente torna-se aplicável para um novo contexto.

- Atenção: estes alunos podem apresentar problemas significativos de atenção e baixo nível de concentração em uma situação de aprendizagem formal.

- Motivação: alguns destes alunos não se sentem motivados espontaneamente, exigindo a mediação do professor para se envolverem com as atividades, principalmente as que requerem um maior nível de dificuldade e que não tem uma função social imediata e clara.

Apesar destas dificuldades por parte de pessoas com deficiência intelectual, isso não significa necessariamente que elas são incapazes de aprender matemática (CARMO, 2012). 
Estas pessoas não são incapazes de aprender matemática (CARMO, 2012), mas sabe-se muito pouco sobre os déficits cognitivos subjacentes ao seu baixo desempenho nessa área (BRANKAER; GHESQUIÈRE; DE SMEDT, 2011; CARMO, 2012).

A matemática que deve ser ensinada para estas pessoas, deve servir como ferramenta nas atividades cotidianas e para a solução de problemas, focada na compreensão de conceitos básicos, como contagem, numeração, adição, subtração, multiplicação e divisão (JANSEN; DE LANGE; VAN DER MOLEN, 2013; CHUNG; TAM, 2005; CARMO, 2012).

Segundo Rossit (2003) e Carmo (2012), para que o ensino da matemática se torne efetivo, deve-se priorizar a avaliação do repertório de entrada para identificar as habilidades presentes e, em seguida, devem ser estabelecidos os comportamentos alvos a serem ensinados e selecionar os procedimentos de ensino disponíveis.

De maneira geral, o método de ensino convencional de matemática utiliza estratégias que implicam em somente ouvir e ler o problema, anotar a operação, e fazer o cálculo (CHUNG; TAM, 2005). Porém, para estes autores, esta instrução convencional assume a caracterização dos alunos com deficiência intelectual como aprendizes passivos, e não tenta preencher as lacunas na compreensão conceitual dos princípios do pensamento matemático subjacente ou desenvolver habilidades de resolução de problemas.

Para que o ensino de conceitos matemáticos seja efetivo, recomenda-se, que o primeiro passo seja o levantamento de repertório do aluno (CARMO, 2012). Em seguida, o autor recomenda que se elaborem unidades pequenas de ensino, descrevendo um repertório simples a ser ensinado, passo a passo, aumentando gradativamente as exigências de aprendizagem ao aluno, sempre iniciando com etapas fáceis, garantindo os pré-requisitos para o ensino de um novo conceito. Por fim, recomenda-se que devam estabelecer situaçóes de observação e imitação de modelos, e proporcionar manipulação de materiais concretos, usando para tal, jogos e brincadeiras, para reforçar o conteúdo que se está pretendendo ensinar, tentando-se ao mesmo tempo, relacionar o conteúdo matemático às situaçóes cotidianas do aluno (CARMO, 2012).

Desse modo, esta pesquisa surge da necessidade de uma educação na qual todas as pessoas com necessidades especiais (ou não) teriam acesso à escola, de maneira igualitária, cuja meta principal seria oferecer oportunidades iguais para que todos desenvolvam seu potencial (ROSSIT; ZULIANI, 2003; COSTA, 2013).

A partir disso surgem as questóes de pesquisa:

Qual o conteúdo matemático que vem sendo abordado nos estudos empíricos? Quais materiais e estratégias de ensino têm sido utilizados para ensinar matemática a estes alunos?

\section{Objetivo}

Verificar, na literatura científica nacional e internacional, entre os anos de 2003 a 2014, o conteúdo matemático dos estudos empíricos, bem como estratégias bem-sucedidas de inclusão e de avaliação de repertório de entrada para esta população. 


\section{Método}

Foi realizada uma revisão sistemática da literatura (HOHENDORFF, 2014), baseada em artigos empíricos publicados entre janeiro de 2003 e dezembro de 2014.

Tais estudos estavam indexados nas bases de dados da Biblioteca Virtual SciELO Brasil (Scientific Electronic Library Online), EBSCOhost (Academic Search Premier) e ERIC (Education Resources Information Center), todas acessadas via base de dados da CAPES (Coordenação de Aperfeiçoamento de Pessoal de Nível Superior). Além disso, foram consultados os periódicos especializados, Revista Brasileira de Educação Especial e Revista de Educação Especial.

A busca foi efetuada a partir de descritores representativos à temática de investigaçáo, que foram utilizados isoladamente e em associação, em português e em inglês: 'habilidades matemáticas' \& 'deficiência intelectual'; 'matemática' \& 'deficiência intelectual'.

Para análise dos dados, foram produzidas categorias sobre características e conteúdos presentes nos artigos, baseados em Silveira, Enuno e Rosa (2012).

\section{Resultados E discussótes}

\subsection{Seleçáo dos ARTigos}

A partir da busca efetuada com os descritores supracitados, foram retornados 37 artigos.

Inicialmente, foram retirados os estudos repetidos e os que tratavam de estudos teóricos. Em seguida, foi realizada a leitura dos títulos e resumos de todos os artigos, para eliminar trabalhos destoantes da temática preterida, além de serem baixados somente os que estavam disponíveis na íntegra nas bases de dados citadas. Assim, chegou-se a um total de 13 artigos, os quais foram lidos integralmente.

\subsection{ANÁlISE E DISCUSSÃo DOS DADOS}

Os resultados provenientes dos artigos foram agrupados em três grandes temas: conteúdo matemático, repertório de entrada e estratégias de ensino.

\subsection{ConTeÚdo MATEMÁtico}

Os conteúdos matemáticos dos trabalhos selecionados podem ser agrupados em três blocos: 1) habilidades pré-aritméticas (tamanho - grande, pequeno, maior, menor, igual; posição - direita, esquerda, dentro, fora, ao lado; direção - para frente, para trás; tempo - antes, depois, noite, dia); 2) habilidades aritméticas (adição, subtração, multiplicação e divisão); e 3 ) numeração, contagem e noções de quantidade. Tais conteúdos matemáticos, de acordo com Brasil (1997), são caracterizados como matemática básica, fazendo parte do currículo regular de ensino da Educação Infantil e do Ensino Fundamental I ( $1^{\circ}$ a $5^{\circ}$ ano).

O quadro a seguir, traz uma visualização geral das produçóes encontradas com pessoas com Deficiência Intelectual (DI): 


\begin{tabular}{|c|c|c|c|}
\hline Referência & País & Participantes & Habilidade \\
\hline Rossit e Zuliani (2003) & Brasil & $\begin{array}{l}\text { Dez adultos com DI, de } 18 \text { a } \\
32 \text { anos }\end{array}$ & $\begin{array}{l}\text { Atividades com números, } \\
\text { contagem e adição, usando } \\
\text { moedas }\end{array}$ \\
\hline $\begin{array}{l}\text { Bashash, Outhred e Bochner } \\
(2003)\end{array}$ & Austrália & $\begin{array}{l}30 \text { pessoas com DI moderada, } \\
\text { entre } 7 \text { e } 18 \text { anos }\end{array}$ & Contagem e número \\
\hline Chung e Tam (2005) & $\begin{array}{l}\begin{array}{l}\text { Hong Kong } \\
\text { (China) }\end{array} \\
\end{array}$ & $\begin{array}{l}30 \text { estudantes com DI leve, entre } \\
9 \text { e } 12 \text { anos }\end{array}$ & Adição e subtração \\
\hline Rietveld (2005) & Nova Zelândia & $\begin{array}{l}3 \text { crianças com Síndrome de } \\
\text { Down, entre } 5 \text { e } 6 \text { anos }\end{array}$ & Atividades com números \\
\hline $\begin{array}{l}\text { Escobal, Rossit e Goyos } \\
(2010)\end{array}$ & Brasil & $\begin{array}{l}\text { Dois adultos com DI, com } 16 \text { e } \\
20 \text { anos }\end{array}$ & Contagem e nomeação \\
\hline $\begin{array}{l}\text { Van Luit e Van der Molen } \\
\text { (2011) }\end{array}$ & Holanda & $\begin{array}{l}70 \text { crianças com DI leve, com } \\
\text { idade média de } 9 \text { anos }\end{array}$ & Nomeação de números \\
\hline $\begin{array}{l}\text { Brankaer, Ghesquiere e De } \\
\text { Smedt (2011) }\end{array}$ & Bélgica & $\begin{array}{l}26 \text { com DI leve e } 52 \text { com } \\
\text { desenvolvimento típico, de } 8 \text { a } \\
11 \text { anos }\end{array}$ & $\begin{array}{l}\text { Conceitos de maior e menor, } \\
\text { adiçáo, subtraçấo }\end{array}$ \\
\hline Rosso e Dorneles (2012) & Brasil & $\begin{array}{l}\text { Dois estudantes, um com Sín- } \\
\text { drome do X-Frágil e outro com } \\
\text { Síndrome de Prader-Willi, } 10 \\
\text { anos de idade }\end{array}$ & $\begin{array}{l}\text { Atividades com números e } \\
\text { contagem }\end{array}$ \\
\hline $\begin{array}{l}\text { Brankaer, Ghesquiere e De } \\
\text { Smedt (2013) }\end{array}$ & Bélgica & $\begin{array}{l}31 \text { com DI leve, e dois grupos } \\
\text { controle de } 31 \text { cada, de } 8 \text { a } 11 \\
\text { anos }\end{array}$ & $\begin{array}{l}\text { Magnitude numérica (maior/ } \\
\text { menor). }\end{array}$ \\
\hline $\begin{array}{l}\text { Jansen, De Lange e Van der } \\
\text { Molen (2013) }\end{array}$ & Holanda & $\begin{array}{l}58 \text { adolescentes na fronteira da } \\
\text { DI, entre } 12 \text { e } 15 \text { anos de idade }\end{array}$ & $\begin{array}{l}\text { Adiçãa, subtração, multipli- } \\
\text { cação e divisão }\end{array}$ \\
\hline Malaquias at. al. (2013) & Brasil & $\begin{array}{l}15 \text { alunos com DI, entre } 13 \text { e } \\
22 \text { anos }\end{array}$ & Habilidades pré-aritméticas \\
\hline $\begin{array}{l}\text { Brito, Campos e Romanatto } \\
\text { (2014) }\end{array}$ & Brasil & $\begin{array}{l}\text { Três alunos com desenvolvimen- } \\
\text { to típico do Ensino de Jovens e } \\
\text { Adultos (EJA), dois alunos da } \\
\text { EJA com DI, e uma professora, } \\
\text { com idades variando entre } 31 \text { e } \\
75 \text { anos }\end{array}$ & $\begin{array}{l}\text { Contagem, quantificaçáo, } \\
\text { sistema de numeração deci- } \\
\text { mal e as operaçóes de adição } \\
\text { e subtração }\end{array}$ \\
\hline Yakubova e Bouck (2014) & EUA & $\begin{array}{l}\text { Cinco alunos com DI leve, todos } \\
\text { com } 11 \text { anos de idade }\end{array}$ & Adição e subtração \\
\hline
\end{tabular}

Quadro 1 - Lista dos estudos selecionados

A partir da organização das produções listadas no Quadro 1, pode-se destacar a fragilidade do estudo na área, uma vez que no período pesquisado, foram encontrados 13 trabalhos não distribuídos regularmente, demonstrando lacunas nos anos de 2004, 2006, 2007, 2008 e 2009.

Agora, iniciando-se as análises pelo tópico referente às habilidades pré-aritméticas, que formam a base para a aprendizagem da aritmética, tais como noçóes de maior/menor, mais/menos, grande/pequeno, primeiro/último, antes/depois, início/meio/fim, perto/longe (CARMO, 2012). Além disso, é relevante o ensino de discriminação de numerais e de quantidades de um a dez, conservação de quantidades discretas e de quantidades contínuas, equivalência numeral-quantidade e equivalência quantidade-quantidade (CARMO, 2012). 
Concomitante e continuamente, são realizadas as análises referentes às habilidades aritméticas (adição, subtração, multiplicação e divisão).

Iniciando-se pelo estudo de Malaquias et al. (2013), que teve o objetivo de investigar a viabilidade do jogo VirtualMat para o ensino de conceitos lógico-matemáticos para 15 alunos com deficiência intelectual, entre 13 e 22 anos, foram aplicadas atividades que envolviam conceitos de habilidades pré-aritméticas, chegando-se a conclusão de que, através do jogo computacional, ficou demonstrado o aprendizado destas habilidades, permitindo o desenvolvimento das mesmas em uma forma lúdica e ativa, indo ao encontro de estratégias citadas por Carmo (2012).

Outro estudo que trabalha parte de habilidades pré-aritméticas (conceitos de maior, menor e igual) foi o de Rossit (2003), que trabalhou com 11 participantes com deficiência intelectual, entre 9 e 32 anos, em um teste desenvolvido pela própria autora. Rossit (2003) ressalta que foram apenas quatro participantes que tiveram desempenho inferior a 80\%, mostrando, de maneira geral, bom desempenho nestas habilidades. A autora trabalhou o conceito de adição, focado no uso desta em atividades de vida autônoma, solicitando ao participante que, usando notas e moedas (dinheiro), ele precisaria chegar a um determinado valor. Como resultados, dez participantes tiveram desempenho inferior a $80 \%$ de aproveitamento, sendo que cinco deles tiveram desempenho zero, ou seja, mostrando, de maneira geral, grande dificuldade no desempenho desta atividade que envolvia conceito de adição.

Nos estudos de Brankaer, Ghesquiere e De Smedt (2011, 2013), foram trabalhados os conceitos de magnitude numérica (maior/menor), por meio da comparaçáo entre o desempenho de crianças e adolescentes com deficiência intelectual e com desenvolvimento típico. No estudo de Brankaer, Ghesquiere e De Smedt (2011), participaram 26 crianças com deficiência intelectual leve, e dois grupos controle de 26 crianças com desenvolvimento típico cada. Como resultado, concluiu-se que os participantes com DI têm maior dificuldade com representação de magnitude numérica (maior e menor) do que aqueles com desenvolvimento típico.

No estudo de Brankaer, Ghesquiere e De Smedt (2013), participaram 31 adolescentes com DI leve, e dois grupos controle de 31 adolescentes com desenvolvimento típico cada, com idades entre 12 e 15 anos. Chegou-se ao resultado de que os participantes com DI tiveram um desempenho inferior do que seus pares de mesma idade cronológica, nas tarefas de comparação de magnitude, sugerindo deficiências na capacidade dessas crianças para representá-las. Eles também tiveram um desempenho inferior na memória de trabalho em relação aos seus pares com desenvolvimento típico.

Ambos os estudos usaram o Tempo Test Arithmetic, constituído por 40 problemas com atividades das quatro operações (soma, subtração, multiplicação e divisão), adaptado, com a aplicação de atividades de adição e subtração (DE VOS, 1992; BRANKAER, GHESQUIERE; DE SMEDT, 2011).

O estudo de Jansen, De Lange e Van der Molen (2013), cujo objetivo foi melhorar habilidades de matemática em adolescentes na fronteira da DI (quociente intelectual entre 5585), utilizando treinamento computadorizado de matemática, trabalhou com os conteúdos de adição, subtração, multiplicação e divisão, com 58 adolescentes, entre 12 e 15 anos. As autoras utilizaram o Tempo Test Automatiseren, que é um instrumento independente, muitas vezes usa- 
do para monitorar o desempenho de matemática de crianças holandesas com desenvolvimento típico (DE VOS, 2010; JANSEN; DE LANGE; VAN DER MOLEN, 2013). O teste é constituído de partes referentes à adição, subtração, multiplicação e divisão, nas quais cada operação tinha 50 problemas.

A partir dos resultados, De Lange e Van der Molen (2013) concluem que as habilidades matemáticas podem melhorar, se for feito um esforço na realização de atividades. Também, segundo as autoras, houve uma melhora considerável nas habilidades de adição e subtração, por parte dos adolescentes na fronteira da DI, apesar do desempenho médio não diferirem muito em relação aos adolescentes com desenvolvimento típico.

Chung e Tam (2005) examinaram os efeitos de abordagens tradicional e cognitiva para o ensino de 30 alunos com deficiência intelectual leve, entre oito e 12 anos, para resolver problemas matemáticos, aplicando atividades de adiçáo e subtração, retiradas de livros didáticos do ensino fundamental. Como resultado, os autores dizem que os alunos se beneficiaram das estratégias cognitivas (a leitura do problema em voz alta, seguida de explicação, visualização, cálculo de soma e subtração, e verificação da resposta), para a resolução de problemas corretamente.

Em seguida, destaca-se o estudo de Brito, Campos e Romanatto (2014), que teve o objetivo de conhecer as práticas de um professor da EJA (Ensino de Jovens e Adultos) para o ensino de habilidades matemáticas (contagem, quantificação, sistema de numeração decimal e operaçóes de adição e subtração) a três alunos com desenvolvimento típico e dois alunos com DI, com idades variando entre 31 e 75 anos. Segundo os autores, o estudo foi realizado em uma sala do EJA, onde foram feitas observaçôes e intervençôes mediante aplicação de atividades de ensino por meio de jogos. Como resultados, os autores destacam que ficou demonstrado o aprendizado destas habilidades matemáticas, permitindo seu desenvolvimento de forma lúdica e ativa.

O estudo de Yakubova e Bouck (2014), que teve o objetivo de examinar a eficácia das calculadoras científicas e gráficas como ferramentas educacionais para resolver problemas de computação e subtração de palavras para cinco alunos com DI leve. Como resultados, as autores detectaram uma melhora no desempenho dos alunos em adição e subtração, com a diminuição no tempo de realização das operaçōes.

Por último, passa-se aos estudos ligados à temática referente à numeração e contagem, que, para Rosso e Dorneles (2012), inicia-se na Educação Infantil, sendo fundamental para a aprendizagem de conhecimentos matemáticos posteriores. Para Lorena, Castro-Caneguim e Carmo (2013), a contagem envolve as habilidades de produçáo de cadeia verbal numérica (recitação da sequência dos nomes dos números em uma dada ordem estável, como um, dois, três, quatro...). Também, continuam, envolvem as habilidades de estabelecimento de relação termo a termo, que é a correspondência um para um, ou seja, cada nome de número da sequência numérica verbal corresponde a um e somente um elemento da coleção a ser contada.

Já o conceito de número é entendido como uma rede de relaçóes equivalentes entre numerais (números impressos, quantidades e números falados correspondentes, que passam a fazer parte de uma só classe, de tal forma que, por exemplo, o numeral ' 3 ', três bolinhas e a palavra falada "três" se referem ao mesmo evento numérico). Quantidade, por sua vez, envolve o conceito de número, como as relaçóes de equivalência entre numeral e quantidade (CARMO, 2012). 
Rosso e Dorneles (2012) investigaram os princípios de contagem numérica em dois estudantes com dez anos de idade e deficiência intelectual (um com Síndrome do X-Frágil e outro com Síndrome de Prader-Willi). Como resultado, os autores afirmam que o princípio da correspondência um a um mostra-se em desenvolvimento mais lento nos participantes que nas crianças com desenvolvimento típico na mesma faixa etária.

No estudo de Rossit e Zuliani (2003), que avaliou o ensino de relaçóes condicionais entre componentes numéricos e numerais impressos, utilizando moedas, com dez adultos com DI, entre 18 e 32 anos, foram usadas atividades com números, contagem e adição. Os resultados mostram a eficácia do ensino, visto a aquisição de habilidades em tempo reduzido. Contudo, para as autoras, ainda há muito que se investigar com relação ao processo de ensino-aprendizagem de comportamentos básicos para pessoas com necessidades especiais.

Nessa mesma linha, Escobal, Rossit e Goyos (2010) investigaram o processo de aquisição do conceito de número por pessoas com DI, de 16 a 20 anos, utilizando atividades com números, contagem e nomeação, por meio do uso do Programa Computacional Mestre (ELIAS; GOYOS, 2010). Como resultados, o estudo mostrou a eficácia do currículo informatizado para a aquisição do conceito de número em um curto espaço de tempo.

Rietveld (2005), por sua vez, investigou a entrada de três meninos com Síndrome de Down (entre cinco e seis anos) na escola, e a aprendizagem matemática deles. Como resultado, o autor fala que as barreiras à aprendizagem destas crianças foram evidentes em todos os contextos, principalmente pela falta de acompanhamento por parte dos professores. Os dados do estudo sugerem a necessidade dos professores em adotar um papel como mediadores da aprendizagem, em vez de aplicadores de conteúdo.

Bashash, Outhred e Bochner (2003) investigaram as habilidades matemáticas de contagem e número, e as estratégias utilizadas para o ensino de alunos de sete a dezoito anos, com deficiência intelectual moderada. Foi usado o modelo número-palavra, compreensão e uso de palavras do número pelos alunos em quatro situações (sequência, contagem, cardeais e símbolos). Os resultados mostraram uma diferença significativa entre os três grupos de idade (7-11, 12-15, 16-18) em todas as habilidades numéricas avaliadas. Especificamente, o padrão de aprendizagem para a sequência de palavra-número e os tipos de erros de correspondência feitos foi semelhante aos de alunos com desenvolvimento típico.

Por fim, Van Luit e Van der Molen (2011) examinaram se uma intervenção com o número de nomes na forma coreana influencia a aquisição do conceito de número por 70 alunos com DI leve. Para estes autores, o nosso sistema de numeração, o hindu-arábico, é o mais comumente aceito em todo o mundo, mas a maneira pela qual os números são nomeados difere em cada língua. No holandês, o número 27, por exemplo, é pronunciado como "Zeven en Twintig" (7 e 20). Por outro lado, linguagens baseadas no antigo chinês, como o idioma coreano, pronunciar o número 27 como 'dois-dez-sete'. Neste último modo de nomear, o número dez emerge claramente; o valor lugar de cada dígito dentro de um número é evidente. Em contraste como, por exemplo, as crianças de língua holandesa e de língua inglesa, as asiáticas não têm que conhecer os diferentes nomes dos números entre 10 e 20, como "onze" ou "dezoito”, em vez disso, eles aprendem números pelo mesmo sistema regular e lógico: 'um-dez-um' ou 'um-dez-oito'. 
Van Luit e Van der Molen (2011) utilizaram o Number Insight Test (NIT), que se baseia na avaliação da compreensão dos números, falados e escritos, e representação de quantidade (VAN LUIT, 2007). Os resultados indicaram um efeito positivo deste método alternativo. Entretanto, os participantes não conseguiram fazer generalização para números de 21 a 100 . Assim, o método coreano pareceu ser um caminho promissor para ensinar os alunos com deficiência intelectual leve.

Nota-se que, em todos os trabalhos apresentados, são ensinados conteúdos a participantes com deficiência intelectual que devem servir como ferramenta nas atividades cotidianas e para a solução de problemas, focada na compreensão de conceitos básicos, indo de acordo com Jansen, De Lange e Van Der Molen (2013), Chung e Tam (2005), Rosenblum e Herzberg (2011) e Carmo (2012).

Com relação à construção do conhecimento matemático, os conteúdos de pré-aritmética, numeração e contagem parecem estar presentes antes da criança com desenvolvimento típico entrar na escola (ROSSIT; ZULIANI, 2003). Este fato pode ser evidenciado no desempenho em atividades que requerem esses conceitos, como no caso de contar nos dedos da mão a quantidade correspondente à idade ou o uso da contagem nas brincadeiras infantis, continuando tanto no ensino formal, oferecido pela escola, como no ensino informal (ROSSIT; ZULIANI, 2003). Contudo, continuam as autoras, pessoas com necessidades especiais podem passar pelo período de escolarização sem alcançar o domínio das habilidades matemáticas básicas, e podem chegar à idade adulta sem os pré-requisitos para desempenhar, de forma independente, diversas atividades, ou mesmo, podendo ser aprendidas de forma inapropriada, ou até, não serem aprendidas.

Porém, dentre os conceitos matemáticos básicos, não foi encontrado nenhum trabalho que ensinasse geometria elementar, tanto da geometria plana como espacial, tais como reta, plano, ângulo, área, volume e medidas (LORENZATO, 1995; MOURA; LORENZATO, 2009).

\subsection{RePERTório DE ENTRADA}

Segundo Carmo (2012), para que o ensino da matemática se torne efetivo, deve-se priorizar a avaliação do repertório de entrada para identificar as habilidades presentes. Para o autor, a partir dos dados obtidos nessa avaliação, devem ser estabelecidos os comportamentos alvos a serem ensinados, e selecionados os procedimentos de ensino disponíveis.

Tal fato se torna evidente no estudo de Escobal, Rossit e Goyos (2010), que ressalta que a avaliação cuidadosa do repertório de entrada forneceu os subsídios para o planejamento de ensino direcionado às necessidades individuais. Para eles, a análise das porcentagens de erros, possibilitou a programação do ensino específico para as relaçóes ausentes ou insuficientes, sendo fundamental para o aprendizado dos participantes com deficiência intelectual.

Rossit (2003), avaliando os comportamentos matemáticos básicos presentes no repertório de 11 participantes com deficiência intelectual, chegou a conclusão que, ao identificar as habilidades em que os participantes apresentaram mais erros, criou condiçóes para o planejamento de atividades de ensino das habilidades específicas para as quais os participantes apresentaram maior dificuldade. 
Nos estudos de Brankaer, Ghesquiere e De Smedt $(2011$, 2013), foram trabalhados os conceitos de magnitude numérica (maior/menor), usando o Tempo Test Arithmetic como pré-teste, constituído por 40 problemas com atividades crescentes das quatro operaçóes, mas adaptados, com a aplicação de atividades de adição e subtração. $\mathrm{O}$ uso do Tempo Test Arithmetic como pré-teste foi útil para a avaliação do repertório de entrada e para identificar as habilidades presentes.

No mesmo sentido, o estudo de Jansen, De Lange e Van der Molen (2013) teve o objetivo de melhorar habilidades de matemática em adolescentes na fronteira da DI. As autoras utilizaram o treinamento computadorizado de matemática com o Tempo Test Automatiseren para monitorar o desempenho de matemática de crianças com desenvolvimento típico, constituído de partes referentes à adição, subtração, multiplicação e divisão.

Fica evidente, de acordo com os estudos citados nesse item, a importância de desenvolver testes para avaliação do repertório de entrada dos alunos e usar os dados obtidos para o planejamento do ensino. Vale ressaltar também que esses testes devem ser aplicados durante todo o processo de ensino, considerando que algumas habilidades podem emergir a partir do ensino de outras habilidades (CARMO, 2012; ESCOBAL, ROSSIT; GOYOS, 2010; ROSSIT, 2003).

\subsection{Estratégias de ENSINO}

Para Carmo (2012), um programa de ensino de repertórios matemáticos para alunos com Deficiência Intelectual deve garantir situaçóes nas quais o aprendiz observe e imite o professor, tendo em vista ser este um modelo a ser seguido. Para este autor, deve-se dar oportunidade para o aluno observar o modelo fazendo algumas operaçóes e, em seguida, imitar o modelo para o estabelecimento de modos eficazes e eficientes de solução de problemas.

No trabalho de Chung e Tam (2005), foram examinados os efeitos de abordagens tradicional e cognitiva para o ensino de 30 alunos com DI leve, entre oito e doze anos, para resolver problemas matemáticos, aplicando atividades de adição e subtração retiradas de livros didáticos do ensino fundamental. Como resultado, os autores dizem que os alunos se beneficiaram das estratégias cognitivas (a leitura do problema em voz alta, seguida de explicação, visualização, cálculo e verificação da resposta) para a resolução de problemas corretamente. Além disso, estratégias cognitivas foram mais eficazes para a generalização do conhecimento e das habilidades para resolver problemas novos do que instruções convencionais (leitura e resolução mecânica das atividades).

O estudo de Brito, Campos e Romanatto (2014), que teve o objetivo de conhecer as práticas de um professor da EJA para o ensino de habilidades matemáticas (contagem, quantificação, sistema de numeração decimal e operaçóes de adição e subtração) a três alunos com desenvolvimento típico e dois com DI, com idades variando entre 31 e 75 anos. Os autores usaram como estratégia para o ensino das habilidades matemáticas três jogos, "Fazendo a Feira”, o "Tabuleiro da adição e da subtração" e "Problemas no trânsito". Neste estudo, ressalta-se que o jogo foi um gerador de situaçóes-problema, e desencadeador da aprendizagem do aluno com DI, além de trazer o fator de motivação para a aprendizagem das habilidades matemáticas (LANA, 2010). 
Quando se tomam os estudos de Rossit (2003), Escobal, Rossit e Goyos (2010), e Malaquias at. al. (2013), percebe-se que todos usaram como estratégia de ensino o recurso computacional para o ensino de habilidades matemáticas a pessoas com DI, a partir as estratégias propostas pelo paradigma da equivalência de estímulos (SIDMAN; TAILBY, 1982) e procedimentos de escolha de acordo com o modelo (do inglês matching-to-sample-MTS). Segundo Stromer, Mackay e Stoddard (1992), o paradigma de equivalência de estímulos é educacionalmente significativo e pode produzir economia na tarefa de ensinar. Os autores identificam três aspectos de economia de ensino gerados pelo paradigma de equivalência de estímulos: 1) a rede de relações condicionais permite que o educador identifique quais relaçôes já estão presentes no repertório do aluno, e quais estão ausentes, e que deveriam ser ensinadas, desta forma não é necessário repetir o ensino das relaçóes já existentes; 2) ao ensinar duas relaçóes de discriminação condicional, outras relaçóes emergem, sem que sejam diretamente ensinadas (mas que precisam ser testadas, para se ter certeza que elas emergiram); e 3) as classes já formadas podem ser expandidas e, para os casos de expansão, não é necessário associar o novo estímulo a todos os outros daquela classe, mas a somente um de seus membros.

O paradigma de equivalência de estímulos propóe estratégias para gerar classes de estímulos equivalentes, compostas por estímulos permutáveis em determinados contextos, especialmente verbais. Os procedimentos de ensino das relaçóes pré-requisitos para a formação de classes equivalentes utilizam contingências de quatro termos. Os quatro termos são: estímulo condicional, estímulo discriminativo, resposta e consequência. Por exemplo, dados dois estímulos de comparação, B1 e B2, a seleção de B1 na presença do estímulo condicional A1 é reforçada e a seleção de B2 na presença de A2 é reforçada, ou seja, ao ouvir "Qual é o número um?”, a seleção de um cartão com o número "1" impresso, em meio a outros cartôes com outros números, é reforçada; ao ouvir "Qual é o número dois?", a seleção de um cartão com o número "2" impresso, em meio a outros cartóes com outros números, é reforçada; e assim por diante.

Para testar se um procedimento de discriminação condicional gerou relaçóes equivalentes, é necessário haver, pelo menos, três conjuntos de estímulos ( $\mathrm{A}, \mathrm{B}$ e $\mathrm{C})$ com três estímulos em cada conjunto (A1, A2, A3, B1, B2, B3, C1, C2 e C3). Os indivíduos podem ser ensinados a selecionar condicionalmente os estímulos comparaçóes do conjunto $\mathrm{B}$ na presença dos estímulos modelos do conjunto $\mathrm{A}$ (relaçóes $\mathrm{AB}$ ) e os estímulos comparaçóes do conjunto $\mathrm{C}$ na presença dos estímulos modelos do conjunto B (relaçóes BC). Após terem sido explicitamente ensinadas as relações entre os seis pares de modelo-comparação (A1B1, A2B2, A3B3, B1C1, B2C2 e B3C3), os indivíduos imediatamente emparelham os estímulos dos conjuntos $\mathrm{A}$ e $\mathrm{C}$ e seis novas relaçóes emergem sem ensino direto (A1C1, A2C2, A3C3, C1A1, C2A2 e C3A3). As 12 relaçóes de estímulos, seis ensinadas e seis emergentes, definem a existência de três classes de estímulos com três membros cada: A1B1C1, A2B2C2 e A3B3C3 (SIDMAN; TAILBY, 1982).

Para verificar se os estímulos são equivalentes, depois de instaladas as discriminações condicionais entre eles são necessários fazer testes das relações emergentes seguindo as três propriedades da definição matemática de equivalência: reflexividade, simetria e transitividade. Reflexividade corresponde à relação condicional entre dois estímulos idênticos (relaçóes AA, $\mathrm{BB}$ e $\mathrm{CC}$ ). Simetria corresponde à relação condicional inversa à ensinada entre dois estímulos diferentes (se $\mathrm{AB}$, então $\mathrm{BA}$ ). Transitividade é a relação condicional entre dois estímulos diferentes não relacionados anteriormente (se $\mathrm{AB}$ e $\mathrm{BC}$, entâo $\mathrm{AC}$ ). Pode-se citar, ainda, o teste que 
envolve duas propriedades combinadas, transitividade e simetria, comumente denominada de teste de equivalência (CA).

O procedimento utilizado na maioria das pesquisas e aplicaçóes práticas para gerar discriminaçóes condicionais é denominado de escolha de acordo com o modelo (do inglês matching-to-sample ou MTS). Numa tentativa típica de MTS, primeiramente, é apresentado um estímulo modelo. Após uma resposta ao estímulo modelo, chamada de resposta de observação (por exemplo, tocá-lo), dois ou mais estímulos comparações são apresentados em locais distintos. Para cada estímulo modelo, um de comparação é designado como positivo ou discriminativo para o reforçamento. Seguindo a resposta do sujeito a um dos estímulos comparações, em tentativas de ensino, consequências programadas são fornecidas. A próxima tentativa é apresentada após um breve intervalo.

Outro estudo que usou a estratégia de ensino computacional, via calculadoras gráficas e científicas, foi realizado por Yakubova e Bouck (2014), que mostraram uma melhora do desempenho dos alunos com este recurso.

Os resultados destes estudos (ROSSIT, 2003; ESCOBAL, ROSSIT; GOYOS, 2010; MALAQUIAS at. al., 2013; YAKUBOVA; BOUCK, 2014), mostraram que a eficácia do currículo informatizado, tendo sido constatada a aquisição de habilidades matemáticas em reduzido período de tempo, além de permitir o desenvolvimento dessas em uma forma agradável e ativa. Destaca-se também nos estudos de Rossit (2003), Escobal, Rossit e Goyos (2010), o uso do sistema computacional Mestre, com bons resultados ao longo do tempo.

\section{CONSIDERAÇÓES FINAIS}

Essa revisão permite inferir que ainda há poucas pesquisas empíricas acerca do ensino de matemática a pessoas com deficiência intelectual.

Todos os estudos encontrados utilizaram somente os conteúdos de pré-aritmética ou de aritmética, caracterizados como matemática básica, fazendo parte do currículo regular de ensino da Educação Infantil e do Ensino Fundamental I, $1^{\circ}$ a $5^{\circ}$ anos (BRASIL, 1997). Vale ressaltar que, para Carmo (2012), este é um conteúdo presente e essencial nas atividades cotidianas e para a solução de problemas.

Porém, dentre os estudos analisados, em nenhum deles foram encontrados os conceitos de geometria, tanto plana como espacial, também fundamentais para a vida cotidiana (LORENZATO, 1995; MOURA; LORENZATO, 2009).

Em relação à avaliação do repertório de entrada, tão importante para que o planejamento e o ensino da matemática se tornem efetivos (CARMO, 2012), encontraram-se apenas cinco trabalhos nos quais esta análise se torna explícita. Quando isto foi feito, os resultados foram positivos, sendo considerado fundamental para o planejamento de um programa de ensino a pessoas com deficiência intelectual. Nos estudos em que foram utilizados os testes holandeses (Tempo Test Automatiseren, Tempo Test Arithmetice, e Number Insight Test), fica implícito a utilidade destes para levantamento de repertório, tanto em pessoas com deficiência intelectual como para aquelas com desenvolvimento típico. 
Quando se fala de estratégias de ensino, o computador surge como uma boa ferramenta para ensino de repertório matemático a pessoas com deficiência intelectual, associada à equivalência de estímulos e à rede de relaçóes entre estímulos e respostas, permitindo produzir e sistematizar procedimentos que tornam mais eficazes o seu ensino para crianças com deficiência intelectual. Outra estratégia usada, em detrimento da tradicional, é a abordagem cognitiva, que deu excelentes resultados, indo ao encontro do detalhamento das etapas de ensino, além do uso de jogos como fator estimulador da aprendizagem (CARMO, 2012).

Dessa forma, o que fica de fato é que há ainda um longo caminho a ser percorrido, em busca de uma escola inclusiva, na qual a pessoa com deficiência intelectual tenha acesso à matemática elementar, fundamental para uma vida autônoma, apesar dos enormes avanços demonstrados nesta revisão.

$\mathrm{Na}$ literatura acerca de habilidades matemáticas em pessoas com deficiência intelectual, notou-se que, antes de introduzir quaisquer habilidades matemáticas elementares, tão importantes para a vida dessas pessoas, faz-se necessário o levantamento do repertório de entrada, ou seja, perceber o que cada uma delas sabe, para somente em seguida realizar o planejamento do ensino.

As pesquisas também mostraram que ainda se faz necessário aprofundar os estudos acerca das operações aritméticas, principalmente multiplicação e divisão, além dos conhecimentos de geometria básica.

Por fim, quanto às estratégias de ensino de matemática para alunos com deficiência intelectual, estas devem garantir situaçóes nas quais o aprendiz observe e imite o professor, tendo em vista ser este um modelo a ser seguido. Dentro das estratégias encontradas na literatura, observou-se promissora a ferramenta computacional, associada à equivalência de estímulos e à rede de relaçóes entre estímulos e respostas, que, por sua vez, vem permitindo produzir e sistematizar procedimentos que tornam mais eficazes o seu ensino para crianças com deficiência intelectual.

\section{REFERÊNCIAS}

AMERICAN PSYCHIATRIC ASSOCIATION et al. Intellectual disability fact sheet-DSM-5. Arlington, VA: American Psychiatric Association, 2013.

BASHASH, L.; OUTHRED, L.; BOCHNER, S. Counting skills and number concepts of students with moderate intellectual disabilities. International Journal of Disability, Development and Education, v.50, n.3, p.325-345, 2003.

BRANKAER, C.; GHESQUIÈRE, P.; DE SMEDT, B. Numerical magnitude processing in children with mild intellectual disabilities. Research in developmental disabilities, v.32, n.6, p.2853-2859, 2011.

BRANKAER, C.; GHESQUIÈRE, P.; DE SMEDT, B. The development of numerical magnitude processing and its association with working memory in children with mild intellectual disabilities. Research in developmental disabilities, v. 34, n. 10, p.3361-3371, 2013.

BRASIL. DEPARTAMENTO DE POLÍTICA DA EDUCAÇÃO FUNDAMENTAL. Referencial curricular nacional para a educação infantil. Brasília, DF: MEC/SEF, 1997. 
BRITO, J.; CAMPOS, J. A. P. P.; ROMANATTO, M. C. Ensino da matemática alunos com deficiência intelectual na educação de jovens e adultos. Revista Brasileira de Educação Especial, v.20, n.4, p.525-540, 2014.

CARMO, J. S. Aprendizagem de conceitos matemáticos em pessoas com deficiência intelectual. Revista de Deficiência Intelectual, v.3, p.43-48, 2012.

CHUNG, K. K. H.; TAM, Y. H. Effects of cognitive-based instruction on mathematical problem solving by learners with mild intellectual disabilities. Journal of Intellectual and Developmental Disability, v.30, n.4, p.207-216, 2005.

COSTA, A. B. Atividades de ensino sobre o conceito de fração para adolescentes com e sem deficiência visual. 2013. 130f. Dissertação (Mestrado em Educação Especial) - Programa de Pós-Graduação em Educação Especial, Universidade Federal de São Carlos, São Carlos, 2013.

CRUZ, P.; BERGAMASCHI, A.; REIS, M. L. M. De olho nas metas 2011: quarto relatório de monitoramento das 5 metas do todos pela educação. São Paulo: Ed. Moderna, 2012.

DE VOS, T. Manual tempotoets automatiseren. Amsterdam: Boom Testuitgevers, 2010.

DE VOS, T. Tempo test arithmetic - manual. Nijmegen: Berkhout, 1992.

ELIAS, N. C.; GOYOS, C. Mestrelibras no ensino de sinais: tarefas informatizadas de escolha de acordo com o modelo e equivalência de estímulos. Das margens ao centro: perspectivas para as políticas e práticas educacionais no contexto da educação especial inclusiva, v.1, p.223-234, 2010.

ESCOBAL, G.; ROSSIT, R. A. S.; GOYOS, C. Aquisição de conceito de número por pessoas com deficiência intelectual. Psicologia em Estudo, v.15, n.3, p.467-475, 2010.

HOHENDORFF, J. Como escrever um artigo de revisão de literatura. In: (Org.). Publicar em psicologia: um enfoque para a revista científica. São Paulo: Associação Brasileira de Editores Científicos de Psicologia / Instituto de Psicologia da Universidade de São Paulo, 2009. p.39-54.

JANSEN, B. R. J.; DE LANGE, E.; VAN DER MOLEN, M. J. Math practice and its influence on math skills and executive functions in adolescents with mild to borderline intellectual disability. Research in developmental disabilities, v.34, n.5, p.1815-1824, 2013.

LANA, A. V. O jogo e a prática pedagógica: o ensino de matemática através de jogos para crianças com dificuldades de aprendizagem. 2010. 146f. 2010. Tese de Doutorado. Dissertação (Mestrado em Educação) - Universidade Federal do Espírito Santo, Vitória, 2010.

LORENA, A. B.; CASTRO-CANEGUIM, J. F.; CARMO, J. S. Habilidades numéricas básicas: algumas contribuiçốes da análise do comportamento. Estudos de Psicologia, v.18, n.3, p.439-446, 2013.

LORENZATO, Sérgio. Por que não ensinar geometria. Educação Matemática em Revista, v.4, p.3-13, 1995.

MALAQUIAS, F. F. O. et al. VirtualMat: A serious game to teach logical-mathematical concepts for students with intellectual disability. Technology and Disability, v.25, n.2, 2013.

MOURA, A. R. L.; LORENZATO, S. O medir de crianças pré-escolares measurement actions of preschool children. p. 7-42. Zetetiké: Revista de Educação Matemática, v.9, p.15-16, 2009.

RIETVELD, C. M. Classroom learning experiences of mathematics by new entrant children with Down syndrome. Journal of Intellectual and Developmental Disability, v.30, n.3, p.127-138, 2005. 
ROSENBLUM, L. P.; HERZBERG, T. Accuracy and techniques in the preparation of mathematics worksheets for tactile learners. Journal of Visual Impairment \& Blindness, v.105, n.7, p.402-413, 2011.

ROSSIT, R. A. S. Matemática para deficientes mentais: contribuiçóes do paradigma de equivalência de estímulos para o desenvolvimento e avaliação de um currículo. 2003. 180f. Tese (Doutorado) Programa de Pós-Graduação em Educação Especial, Universidade Federal de São Carlos, São Carlos, 2003.

ROSSIT, R. A. S.; ZULIANI, G. Repertórios acadêmicos básicos para pessoas com necessidades especiais. Temas em Psicologia, v.11, n.2, p.114-121, 2003.

ROSSO, T. R. F.; DORNELES, B. V. Contagem numérica em estudantes com síndromes de X-Frágil e Prader-Willi. Revista Brasileira de Educação Especial. Marília, v.18, n.2, p.231-244, 2012.

SIDMAN, M.; TAILBY, W. Conditional discrimination vs. matching to sample: An expansion of the testing paradigm. Journal of the Experimental Analysis of behavior, v.37, n.1, p.5-22, 1982.

SILVEIRA, K. A.; ENUMO, S. R. F.; ROSA, E. M. Concepçóes de professores sobre inclusão escolar e interaçóes em ambiente inclusivo: uma revisão da literatura. Revista Brasileira de Educação Especial, Marília, v.18, n.4, p.695-708, 2012.

STROMER, R.; MACKAY, H. A.; STODDARD, L. T. Classroom applications of stimulus equivalence technology. Journal of Behavioral Education, v.2, n.3, p.225-256, 1992.

VAN LUIT, J. E. H. Two tens and seven. More insight in math with Korean number naming. Balans Magazine, v.20, n.4, p.34-35, 2007.

VAN LUIT, J. E. H.; VAN DER MOLEN, M. J. The effectiveness of Korean number naming on insight into numbers in Dutch students with mild intellectual disabilities. Research in developmental disabilities, v.32, n.5, p.1941-1947, 2011.

YAKUBOVA, G.; BOUCK, E. C. Not all created equally: Exploring calculator use by students with mild intellectual disability. Education and Training in Autism and Developmental Disabilities, v.49, n.1, p.111, 2014.

Recebido em: 26/07/2015

Reformulado em: 15/03/2016

Aprovado em: 17/03/2016 
COSTA, A.B.; PICHARILLO, A.D.M.; ELIAS, N.C. 